\title{
A Novel Self-Activated Mechanism for Stable Liquid Transportation Capable of Continuous-Flow and Real-time Microfluidic PCRs
}

\author{
Di Wu ${ }^{\mathbb{D}}$, Bing Shi, Bin Li and Wenming Wu *(D) \\ Changchun Institute of Optics, Fine Mechanics and Physics (CIOMP), Chinese Academy of Sciences, Beijing \\ 100864, China; wudi16@mails.ucas.ac.cn (D.W.); shibing1105@foxmail.com (B.S.); libin17@mails.ucas.ac.cn (B.L.) \\ * Correspondence: wuwm@ciomp.ac.cn; Tel.: +86-431-8670-8159
}

Received: 17 April 2019; Accepted: 27 May 2019; Published: 28 May 2019

check for updates

\begin{abstract}
The self-activated micropump capable of velocity-stable transport for both single-phased plug and double-phased droplet through long flow distance inside 3D microchannel is one dream of microfluidic scientists. While several types of passive micropumps have been developed based on different actuation mechanisms, until today, it is still one bottleneck to realize such a satisfied self-activated micropump for the stable delivery of both single and double-phased liquid inside long microchannel (e.g., several meters), due to the lack of innovative mechanism in previous methods. To solve this problem, in this article, we propose a new self-activated pumping mechanism. Herein, an end-opened gas-impermeable quartz capillary is utilized for passive transport. Mechanism of this micropump is systemically studied by both the mathematical modeling and the experimental verifications. Based on the flow assays, it totally confirmed a different pumping principle in this paper, as compared with our previous works. The $R^{2}$ value of the overall flow rates inside the 3D microchannel is confirmed as high as 0.999 , which is much more homogeneous than other passive pumping formats. Finally, this novel micropump is applied to continuous-flow real-time PCRs (both plug-type and microdroplet-type), with the amplification efficiency reaching 91.5\% of the commercial PCR cycler instrument.
\end{abstract}

Keywords: self-activated fluidic mechanism; microdroplet; 3D microchannel; real-time PCR; house-made setup

\section{Introduction}

Microfluidics is a technique for precise control and manipulation of micro-scale fluids especially under submicron structures [1,2]. As the "heart" of the microfluidic system, micropump plays a significant role in fluidic transport. Due to the important functions of micropumps, many different principles have been designed to automate the transportation.

Depending on the power source, all micropumps can be divided into two categories: Externally-powered and self-powered micropumps. Additionally, all these micropumps have huge application prospects in a wide range of fields, such as drug delivery, blood transport, Chemical and biological analysis, electronic cooling, and so on [3-5].

Externally-powered micropumps (e.g., piezoelectric micropumps [6,7], syringe pump [8-10], thermopneumatic micropump [11], magnetic micropump [12], and acoustic micropump [13,14]) use off-chip power such as electricity, mechanics, magnetism, and acoustic to drive the flow of microfluidic. Benefiting from the stability of the external components and control system, these micropumps can precisely control the speed of the microfluidic. While these micropumps have prominent advantages, they are always giant in instrument size, and thus, it is hard to integrate the external-power micropumps into microfluidic devices to realize a miniaturized all-in-one set-up. 
As a result, several types of self-powered micropumps have also been developed to realize the miniaturization and portability of the total microfluidic set-up. These self-powered micropumps generally use surface tension [15,16], evaporation pressure [17], hydrostatic force [18,19], gas diffusion/permeation [20,21], chemical/enzymatic reaction [20], or biophysics to induce the fluid flow in microfluidic devices.

In the last few years, to maintain and stabilize the flow velocity inside long microchannel (especially $3 \mathrm{D}$ configuration) during long term, we have proposed a self-powered micropump that depends on the permeability coefficient of the silicone or PDMS elastomer to control the flow rate [20,22-24]. While this type of passive micropump displays advantageous performance for stable sample transport, even inside four meters' long microchannel concerning 3D channel-configuration and high temperature microenvironment, there is a non-negligible shortcoming associated with this method, which has not been solved. The self-powered flow is virtually automated by the gas permeability of the silicone [20,23] or PDMS wall [16,24], but the specified permeability coefficient of silicone or PDMS is determined by the size distribution of mini-pores in the elastomer, which usually varies from the fabrication process, curing conditions, and the production batch, and thus increases the difficulty in accurately controlling the passive flow rate.

In this paper, we have proposed a new method to achieve the self-powered micropump, which avoids the disadvantages of the micropump automated by the gas permeability of the silicone [20,24] or PDMS wall $[16,24]$. An end-opened quartz capillary is utilized to replace the end-blocked silicone tube for flow adjustment, which can dramatically increase the volume of liquid transported and the flow-duration. By changing the length and the inner diameter of the quartz capillary that connects the outlet of the microchannel, the speed of the microfluidic can be precisely controlled, and thus qualified for large volume of aqueous/oil phase transport through long microchannel, which was impossible in previous reports. By using the gas permeability of the quartz capillary to control the flow velocity, no liquid will flow into the quartz tube. Benefitting from this improvement, the flow velocity is more uniform and stable. The velocity is systemically studied by adjusting the length and inner diameter of the tail quartz capillary, and the inner pressure of the fluidic conduit as well. Finally, a house-made setup microreactor of continuous-flow real-time PCRs with the sample transported inside 3D spiral chip is realized, and it only relies on a single thermostatic heater for thermal cycle.

\section{Principle}

Figure 1 shows a schematic illustration of the actuation mechanisms of the self-activated micropump applied to both the single-phased and the double-phased transport in the end-opened system. As we can see, it is based on the air permeability from the fluidic conduit to atmosphere through the tail quartz capillary. Thus, the permeation actually relies on the air passing through the hollow channel of the gas-impermeable quartz capillary.

Since the pressure of the compressed air captured inside the fluidic conduit is much higher than the atmospheric pressure, air molecules inside the microchip tend to penetrate to the ambient atmosphere through the hollow channel of quartz capillary. The air permeation only occurs at the outlet of the quartz capillary, which causes a decrease of the air molecules' mole-number in the anterior end of sample plug. Based on Fick's Law, this can be calculated by the following equation.

$$
\begin{gathered}
G_{a}=\frac{D A_{a v e}}{Z}\left(C_{A i a}-C_{A o}\right) \\
C_{A i a}=\frac{P_{a}}{R T} \\
C_{A o}=\frac{P_{A o}}{R T} \\
A_{a v e}=a \pi r_{q}^{2}
\end{gathered}
$$


where $G_{a}$ is diffusion rate, $D$ is equivalent diffusion coefficient, $C_{A i a}$ is inner air molecule concentration in the anterior end of sample plug, $P_{a}$ represents the air pressures in the anterior end of the reagent, $C_{A o}$ is air molecule concentration of ambient atmosphere, $Z$ is diffusion distance and can be represented by the length of quartz capillary, $A_{\text {ave }}$ is the average diffusion area, $r_{q}$ is the diameter of the quartz capillary, $a$ is the correction coefficient between an actual condition and an ideal condition.

The equivalent diffusion coefficient of quartz capillary is only determined by the dimension of its hollow channel, but not variable to the distribution of pore-size in the wall of PDMS or silicone elastomer as previously reported.

As shown in Figure 1a, $P_{p}$ represents the air pressures in the posterior end of the reagent, while $P_{g}$ represents the pressure gradient imposed in the reagent, and can be calculated by the following equation:

$$
P_{a}=P_{p}-P_{g}
$$

For an easier modeling of this new self-activated pumping system, an equivalent condition forms during the microfluidic transport, when the fluidic-flux and air-molecule-penetration is the same with each other, producing the constant pressure $P_{a}$.

If the microchannel is column-configuration, then

$$
v=\frac{\operatorname{Da\pi r} r_{q}^{2}\left(P_{p}-P_{g}-P_{o}\right)}{R T Z r^{2}}
$$

where $v$ is the velocity of the microfluidic, $r$ is the radius of the microchannel. Easily seen, the flow rate decreases if the radius of the microchannel increases.

Since atmospheric pressure $P_{o}$ is constant, and $\left(P_{p}-P_{g}\right)$ is also kept constant, hence the flow rate is $v$ is constant, which can also be represented by the following equation,

$$
v=\frac{\operatorname{Dar}_{q}^{2}}{R T r^{2}} \frac{d P}{d Z}
$$

where $d P / d z$ is the pressure gradient across the hollow channel of the quartz capillary connecting the microchip to the atmosphere.

Based on the above equation, we can predict that the flow velocity increases as the quartz capillary's length $(Z)$, inner diameter $\left(r_{q}\right)$, and inner compressed pressure $\left(P_{a}, P_{p}\right)$ increase.

Both the flow of liquid plug and liquid droplet can be realized by this self-activated micropump. As we can see in Figure 1b, before we set the pressure by pressing the syringe, the air pressure all through the fluidic conduit is the same. After the reagent and the oil phase (HFE 7500) are added into the insert tips of the $5 \mathrm{~mL}$ and $10 \mathrm{~mL}$ syringes, the pressure of each syringes maintains the same during the flow process. If the volume ratio between the $10 \mathrm{~mL}$ and $5 \mathrm{~mL}$ syringes is $\mathrm{n}: 1$, then the velocity ratio between the oil and the reagent is also $\mathrm{n}: 1$. If the velocity of the oil and the reagent are $v_{0}$ and $v_{r}$, it can be calculated that $v_{0}=n v_{r}$. Therefore, the reagent is divided into droplets by the oil, with the micropump here keeping a steady and homogeneous transport velocity when delivering reagents through a very long microchannel.

Noticeably, herein the principal depends on ideal gas but not the actual gas, which can cause a difference between the principle prediction (ideal condition) and measured experimental results (actual condition). In addition, the diffusion mechanism mainly depends on the Fick's Law and thus is only available for very thin capillary. If the diameter of the capillary is huge, e.g., bigger than $100 \mu \mathrm{m}$, then other regularity such as the convection mass transfer will become the dominant effect. Under such conditions, other factors such as Peclet number of air transport can be relatively high (i.e., $\mathrm{Pe}>2$ ), and resulted in relatively higher flow rate than the air diffusion rule here. Since a very high flow rate is non-applicable for the downstream continuous flow PCR filed here, the flow rule activated by the convection mass transfer will not be discussed in this paper. 


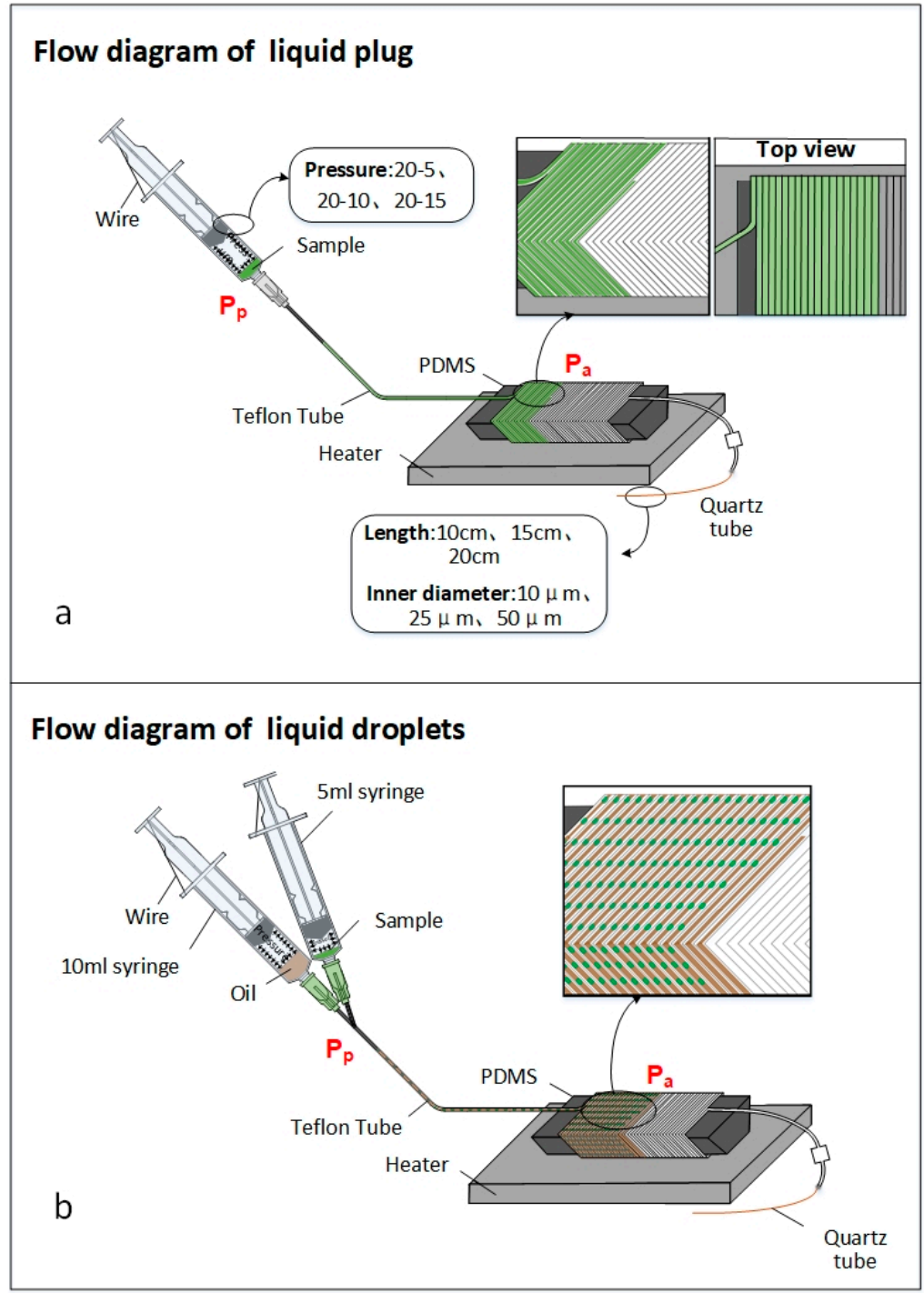

Figure 1. The principle of self-activated micropump applied to the end-opened system, where $P_{p}$ and $P_{a}$ represent the air pressures in the posterior and anterior ends of the reagent. (a) The self-activated plug flow and (b) the self-activated droplet flow.

\section{Experimentation}

\subsection{Flow Experiment}

During the flow analysis, the device set-up consisted of two $20 \mathrm{~mL}$ syringes, one quartz capillary, one clamp, one $27 \mathrm{G}$ needle, one $5 \mathrm{~cm}$-long silicone tube (OD $=3 \mathrm{~mm}, \mathrm{ID}=1 \mathrm{~mm})$, one iron wire and Teflon tube with its inner and outer diameters to be $0.3 \mathrm{~mm}$ and $0.6 \mathrm{~mm}$, respectively. Next, $30 \mu \mathrm{L}$ to $50 \mu \mathrm{L}$ green ink was added to the syringe, and then the Teflon tube was blunt-ended by a clamp. This system was activated by pressing the syringe from the scale $20 \mathrm{~mL}$ to a certain scale, and was fixed by the iron wire. At the end of the system, between the Teflon tube and the quartz capillary, we added a $5 \mathrm{~cm}$-long silicone tube to contain the liquid in case it might flow into the quartz capillary and affected the stability of flow velocity.

After the devices have been installed, the green ink was moved to the pin of the syringe. After removing the clamp, the ink flew spontaneously into the Teflon tube under the consistent pressure inside the system.

We designed three groups of experiments to explore the flow of liquid plug under three different conditions. 
1. By changing the inner diameter of the tail quartz capillary

This experiment was carried out with three different quartz capillaries which had different inner diameters of $10 \mu \mathrm{m}, 25 \mu \mathrm{m}$ and $50 \mu \mathrm{m}$, respectively. To maintain the other parameters the same, the length of the quartz capillary was set to be $10 \mathrm{~cm}$, and the $20 \mathrm{~mL}$ syringe was pressed from the scale of $20 \mathrm{~mL}$ to the scale of $10 \mathrm{~mL}$. Each experiment was operated for three times.

2. By changing the pressure of the syringe

This experiment was carried out by varying the inner pressures of the syringe. The pistons of $20 \mathrm{~mL}$ syringe was pushed from scale $20 \mathrm{~mL}$ to scale $5 \mathrm{~mL}, 10 \mathrm{~mL}$, and $15 \mathrm{~mL}$, respectively. The quartz capillary's inner diameter was $25 \mu \mathrm{m}$, and its length was $15 \mathrm{~cm}$. Each experiment was operated for three times.

3. By changing the length of the tail quartz tube

This experiment was carried out with three different lengths of quartz capillaries, namely $10 \mathrm{~cm}, 15 \mathrm{~cm}$ and $20 \mathrm{~cm}$. And the quartz capillary's inner diameter was $25 \mu \mathrm{m}$. The $20 \mathrm{~mL}$ syringe is pressed from the scale of $20 \mathrm{~mL}$ to $10 \mathrm{~mL}$. Three groups of experiments were operated, with each experiment repeated for three times.

\subsection{Application in Continuous-flow On-chip PCRs}

After the flow experiments, we tested the self-activated micropump system for applications of continuous-flow on-chip PCRs. Altogether, we prepared two sets of experiments to evaluate our system, the reaction of plug-based PCR and the droplets-based PCR.

\section{The plug-based PCR}

The Teflon tube was twined around a trapezoidal PDMS block for 40 rings instead of a syringe. The widths of the top and the bottom surface of the trapezoidal PDMS block are $20 \mathrm{~mm}$ and $10 \mathrm{~mm}$, with the height and length set to be $8.5 \mathrm{~mm}$ and $50 \mathrm{~mm}$, respectively. We cover the block with black tape for the purpose of measuring its surface temperature by IR camera. The $20 \mathrm{~mL}$ syringe is pressed from the scale of $20 \mathrm{~mL}$ to the scale of $10 \mathrm{~mL}$. The quartz capillary's inner diameter is $25 \mu \mathrm{m}$, and its length is $15 \mathrm{~cm}$. All the connections were sealed with silicone adhesive. As shown in Figure 2c, we put a coin at the upper right corner of the device to visually show its total size.

A single heater can achieve the thermal cycle requirement by controlling the temperature gradient of the PDMS block. In order to make sure the temperatures of the upper and lower surfaces were suitable for continuous flow microfluidic PCRs, we used an infrared (IR) camera (Fotric 220, ZXF Laboratory, Allen, TX, USA) to monitor the temperature. The tube wrapped PDMS block was placed on the top of the $95^{\circ} \mathrm{C}$ heater. Through a series of parallel experiments, we adopted to make the height of the PDMS block to be $8.5 \mathrm{~mm}$, so that the temperature of the upper surface reached $60^{\circ} \mathrm{C}$, which is suitable for the PCR reagent.

To prove that the micro-device can be used to continuous flow PCRs, we used a commercial PCR cycler (CFX Connect, Bio Rad, Hercules, CA, USA) as reference. By comparing the product of the two devices, we could verify the function of our system.

The PCR Reagents contained a buffer composed of 1X SYBR Premix Ex Taq II, $0.075 \mathrm{U}_{\mu} \mathrm{L}^{-1} \mathrm{TaKaRa}$ EX Taq, $0.6 \mathrm{mg} \cdot \mathrm{mL}^{-1}$ BSA (AS25483, AMEKO, Dalian, China), $1 \mu \mathrm{M}$ forward and reverse primers, and $0.3326 \mathrm{ng} / \mu \mathrm{L}$ template. The primer sequences were as follows:

\section{5' ACA GAA TCA GGG GAT AAC GCA GGA AAG AAC A 3’ (forward); \\ 5' GTC AGG GGG GCG GAG CCT ATG GAA AAA C 3’ (reverse).}

The gene of pGEM-3Zf (+) fragment was inserted into pUC57-Kan plasmid vector (Genewiz, Suzhou, China) by recombinase, and it was further used as the PCR target. After the PCR reaction, agarose powder (V900510; Sigma-Aldrich, Shanghai, China; www.sigmaaldrich.com, MO; 2\%), DL2000 DNA marker $(50 \times 250 \mu \mathrm{L}$, Peking Jialan Biotechnology Co., Ltd., Beijing, China), $0.5 \times \mathrm{TBE}$ 
buffer (PH1755, Phygene, Fuzhou, China), and Nucleic Acid GelStain (KeyGEN BioTECH, Nanjing, China) were applied to analyze the amplification result.
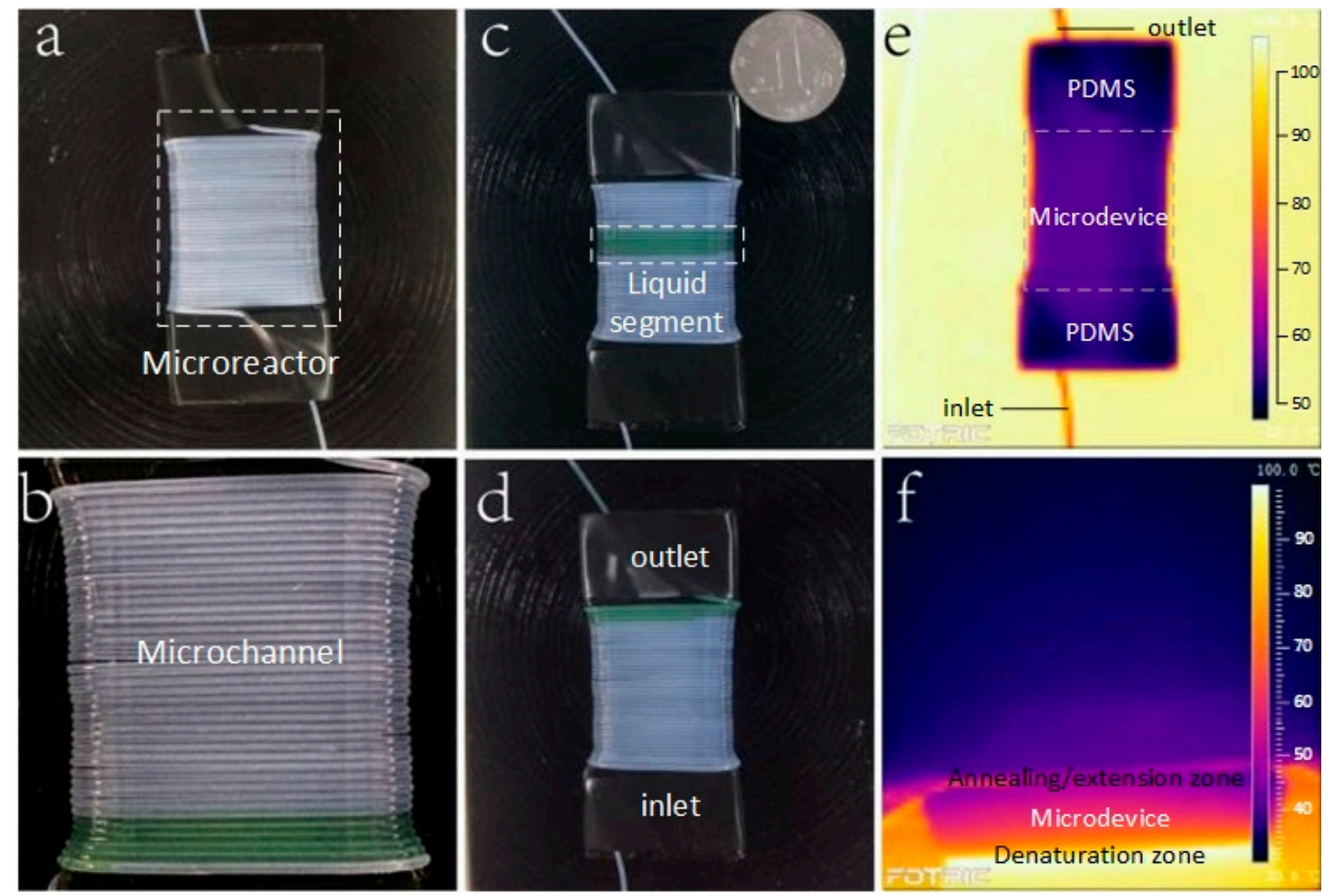

Figure 2. The set-up for plug-based continuous-flow on-chip PCR (a) with the green-color sample flowing at the start (b), the middle (c), and the end (d) of the fluidic conduit. (e) The top view and (f) the side view of the thermal infrared image of the trapezoidal PDMS block.

\section{The droplet-based PCR}

We slightly modify the previous plug-based PCR system as shown in Figure 3. Instead of using one syringe to produce pressure, the droplets generation unit was activated by three pressurized syringes with original volumes of $5 \mathrm{~mL}, 10 \mathrm{~mL}$, and $20 \mathrm{~mL}$, respectively.

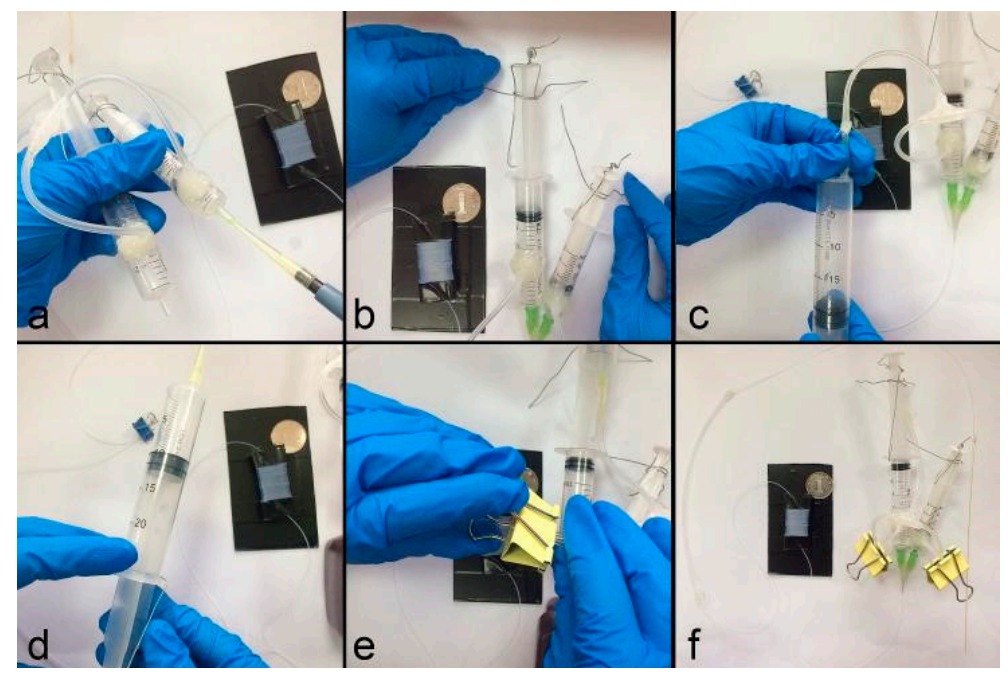

Figure 3. The assembly process for the set-up of the droplet-based continuous-flow on-chip PCR. (a) The injection of the liquid and oil phases; $(\mathbf{b}, \mathbf{c})$ the joint of the device; $(\mathbf{d}, \mathbf{e})$ pressure setting; (f) complete assembly of droplet formation system. 
Before assembling the device, two holes were drilled (Jeben, Yongkang, China) at the $1 \mathrm{~mL}$ graduation of the $5 \mathrm{~mL}$ syringe and $4.5 \mathrm{~mL}$ graduation of $10 \mathrm{~mL}$ syringe. Three silicone tubes $(\mathrm{ID}=1 \mathrm{~mm}, \mathrm{OD}=3 \mathrm{~mm}$, length $=50 \mathrm{~mm}$ ) were used to interconnect the $5 \mathrm{~mL}, 10 \mathrm{~mL}$ and $20 \mathrm{~mL}$ syringes by one T-connect $(\mathrm{ID}=1 \mathrm{~mm}, \mathrm{OD}=1.6 \mathrm{~mm})$. The oil-phase and the aqueous-phase were added to $10 \mathrm{~mL}$ and $5 \mathrm{~mL}$ syringes, which were then connected to the inlet of Teflon tube by two $34 \mathrm{G}$ needles, with the junction sealed by hot melt adhesive. The pistons of the $5 \mathrm{~mL}$ syringe and the $10 \mathrm{~mL}$ syringe were fixed at the scale of $2 \mathrm{~mL}$ and $9 \mathrm{~mL}$ by iron wires. Next the $20 \mathrm{~mL}$ syringe was pushed from the scale of $20 \mathrm{~mL}$ to $10 \mathrm{~mL}$, to produce an inner pressure and fixed by a third iron wire. Then, we used two clamps to blunt the two silicone tubes between the T-connect and $5 \mathrm{~mL}, 10 \mathrm{~mL}$ syringes. The two syringes can be placed either vertically or obliquely to make both the oil and the reagent flow downward spontaneously [25].

As shown in Figure $4 \mathrm{a}$, the droplets were automatically generated and transported inside the Teflon tube. The same PCR chip as previous plug experiment was used, with the length and inner diameter of the tail quartz capillary set to be $20 \mathrm{~cm}$ and $25 \mu \mathrm{m}$, respectively.

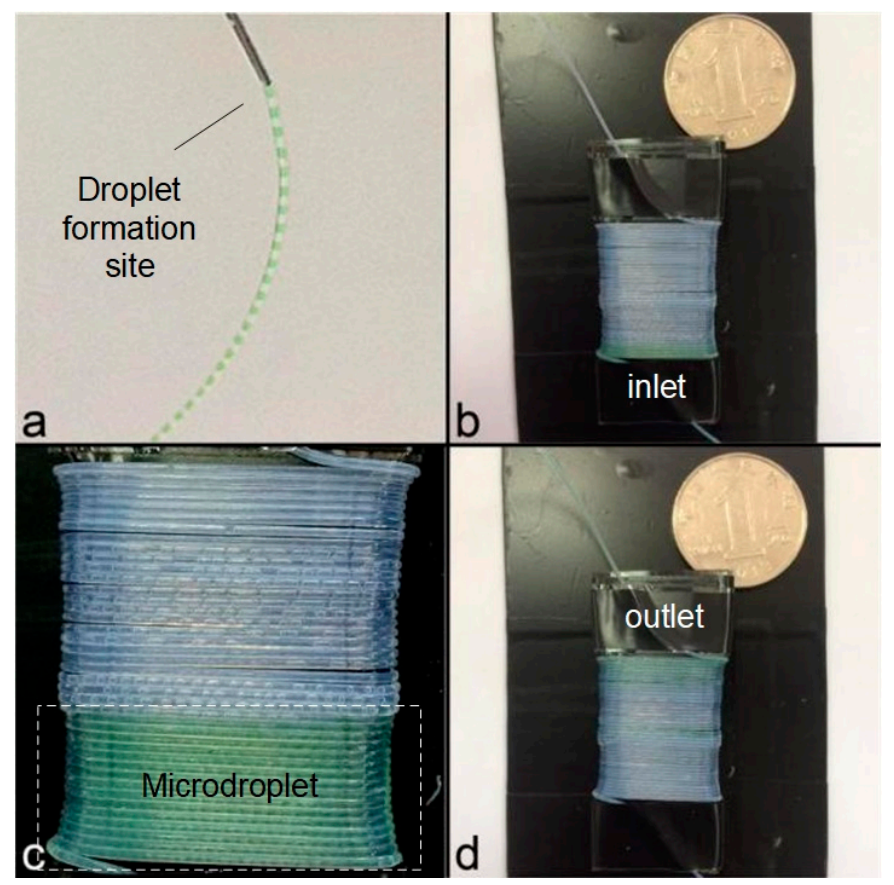

Figure 4. The droplet-based continuous-flow on-chip PCR with the green-color sample flowing at the inlet (a), the start (b), the middle (c), and the end (d) of the fluidic conduit.

We used a single heater to achieve the temperature requirement of the PDMS block wrapped with Teflon tube. The Fluorinated oil (HFE 7500) was used as the oil phase. The other reagents were the same as the plug-based PCR experiment.

\subsection{Real-time Fluorescence Detection}

To prove the microdevice can be used to continuous-flow real-time PCRs, a commercial real-time qPCR (CFX Connect, Bio Rad) was used as reference. By comparing the fluorescence intensity and the products gained from the two devices, the performance of the micro-device could be verified.

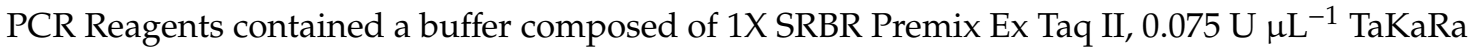
EX Taq, $0.3 \mathrm{mg} \cdot \mathrm{mL}^{-1}$ BSA (AS25483, AMEKO), $1 \mu \mathrm{M}$ forward and reverse primers, and $10^{8}$ to $10^{5}$ copies $\mu \mathrm{L}^{-1}$ DNA template. The primer sequences were as follows:

5' TAC AGA CAA TCC CCG ACC GA 3' (forward)

5' GCC AAG TGT TAG CCC CAT CC 3' (reverse). 
The gene of H7N9 was inserted into pUC57-Kan plasmid vector (Genewiz, Suzhou, China) by recombinase, which was further used as the PCR target.

The fluorescence detection unit consisted of 48 Watts LEDs array (XPE60W, Cree, NC, USA), a digital camera (Canon EOS 7D, Tokyo, Japan), a $480 \mathrm{~nm}$ and a $520 \mathrm{~nm}$ narrowband filter (Xintian bori, Beijing, China). The $480 \mathrm{~nm}$ narrowband filter was fixed in front of the LEDs array to offer the excitation light, and the $520 \mathrm{~nm}$ narrowband filter was fixed in front of the camera lens. The LEDs array was controlled by cycle relay and lighted for $5 \mathrm{~s}$ every $50 \mathrm{~s}$, and the camera connected to the laptop automatically took photos when the LEDs array was on.

The fluorescence images were captured by aforementioned digital camera, with the parameters set as follows: $\mathrm{F}=2.8, \mathrm{M}=1 / 20$, and $\mathrm{ISO}=2000$. The images obtained was processed by the software Image J (version 1.48, National Institutes of Health, Berlin, Germany), to distinguish the light from surroundings conditions. The grayscale value was regarded as the background noise. By counting the light intensity of the liquid plug in each cycle, the fluorescence intensity curve of PCR amplification could be obtained.

\section{Results and Discussion}

\subsection{Flow Analyses}

As shown in Figure 5a, the total running time were 18,483 s, 1016 s, and 62 s, when the inner diameters of the quartz capillary were respectively $10 \mu \mathrm{m}, 25 \mu \mathrm{m}$, and $50 \mu \mathrm{m}$. When the quartz capillary's inner diameter was $10 \mu \mathrm{m}$, the flow resistance was so significant that severely affected the flow rate, causing the velocity of the liquid was extremely slow, and thus, the retention time increased rapidly after 20 rings. On the contrary, the flow velocity of the system was too fast when the inner diameter of tail quartz capillary was $50 \mu \mathrm{m}$, which was around one or two seconds per circle. But when the inner diameter of the quartz capillary was $25 \mu \mathrm{m}$, the flow velocity and the flow time per circle were suitable for the PCR reagents to react, and the linearity of the curve was also better than the other two, with the $R^{2}$ value calculate to be as high as 0.9994 . The results suggested that the flow rate of liquid could be precisely controlled by changing the inner diameter of the quartz capillary connected to the end of Teflon tube. For tail quartz capillary with the inner diameter of $50 \mu \mathrm{m}$, we estimated both the diffusion mechanism and the convection mass transfer affect the flow, and thus, the flow rate is almost 16 times of the tail quartz capillary with the inner diameter of $25 \mu \mathrm{m}$, much bigger than the prediction only determined by the diffusion mechanism, as discussed in the principle part.

Next, the results in Figure $5 b$ showed the effect of the syringe pressure on flow rate. The inner diameter and length of the quartz capillary used in this experiment were $25 \mu \mathrm{m}$, and $15 \mathrm{~cm}$, respectively. Obviously, the flow velocity of liquids was stable and increased as the pressure increased, and the total running time were $978 \mathrm{~s}, 1686 \mathrm{~s}$ and $3945 \mathrm{~s}$ when the syringe was respectively pushed from the scale of $20 \mathrm{~mL}$ to $5 \mathrm{~mL}, 10 \mathrm{~mL}$ and $15 \mathrm{~mL}$. The results suggested that the flow rate of liquids could be adjusted by changing the pressure of the syringe. Based on the principle, the flow rate increased as the inner pressure of the fluidic conduit increased. When the syringe was respectively pushed from the scale of $20 \mathrm{~mL}$ to $5 \mathrm{~mL}, 10 \mathrm{~mL}$, and $15 \mathrm{~mL}$, the inner pressure can be calculated to be $4 \mathrm{~atm}, 2 \mathrm{~atm}$ and $1.33 \mathrm{~atm}$ if the air was considered to be ideal gas. Correspondingly, the ratio of flow rate between three systems was measured to be 5.37:2.29:1.33, implicating a same change-tread with theoretic prediction. Due to the difference between the ideal gas and the real gas, the difference between principle predication and the experiment assays are inevitable.

Finally, the results in Figure 5c were obtained by analyzing quartz capillaries with the same inner diameter of $25 \mu \mathrm{m}$, but with different lengths of $10 \mathrm{~cm}, 15 \mathrm{~cm}$ and $20 \mathrm{~cm}$, respectively. Obviously, the flow rate of liquid was relatively stable and decreased as the length of the Teflon tube increased. The average running time of the liquid flow were $26 \mathrm{~s}, 42 \mathrm{~s}$, and $53 \mathrm{~s}$, respectively. The results showed that the length has great influence on liquid velocity. As demonstrated in Equation (6) of the principle, longer quartz capillary caused bigger diffusion length (Z), and thus decreased flow rate. Based on Equation (6), 
the velocity ratio of the three systems should be 6:4:3 if the lengths of quartz capillary were set to be $10 \mathrm{~cm}, 15 \mathrm{~cm}$ and $20 \mathrm{~cm}$, respectively. And as we can see in Figure $5 \mathrm{~d}$ the experimental results verified velocity ratio between the three systems should be 6:3.72:2.94, which was close to the novel principal in this paper. It also proved here that the pumping principle was totally different from our previous works relying on the permeability coefficient of the end-blocked gas-permeable silicone or PDMS wall according to the experimental results in Figure 5d. In our previous work, the flow rate is proportional to the tube length that connected to the outlet of fluidic conduit, but herein it displays an inversely proportional relationship.
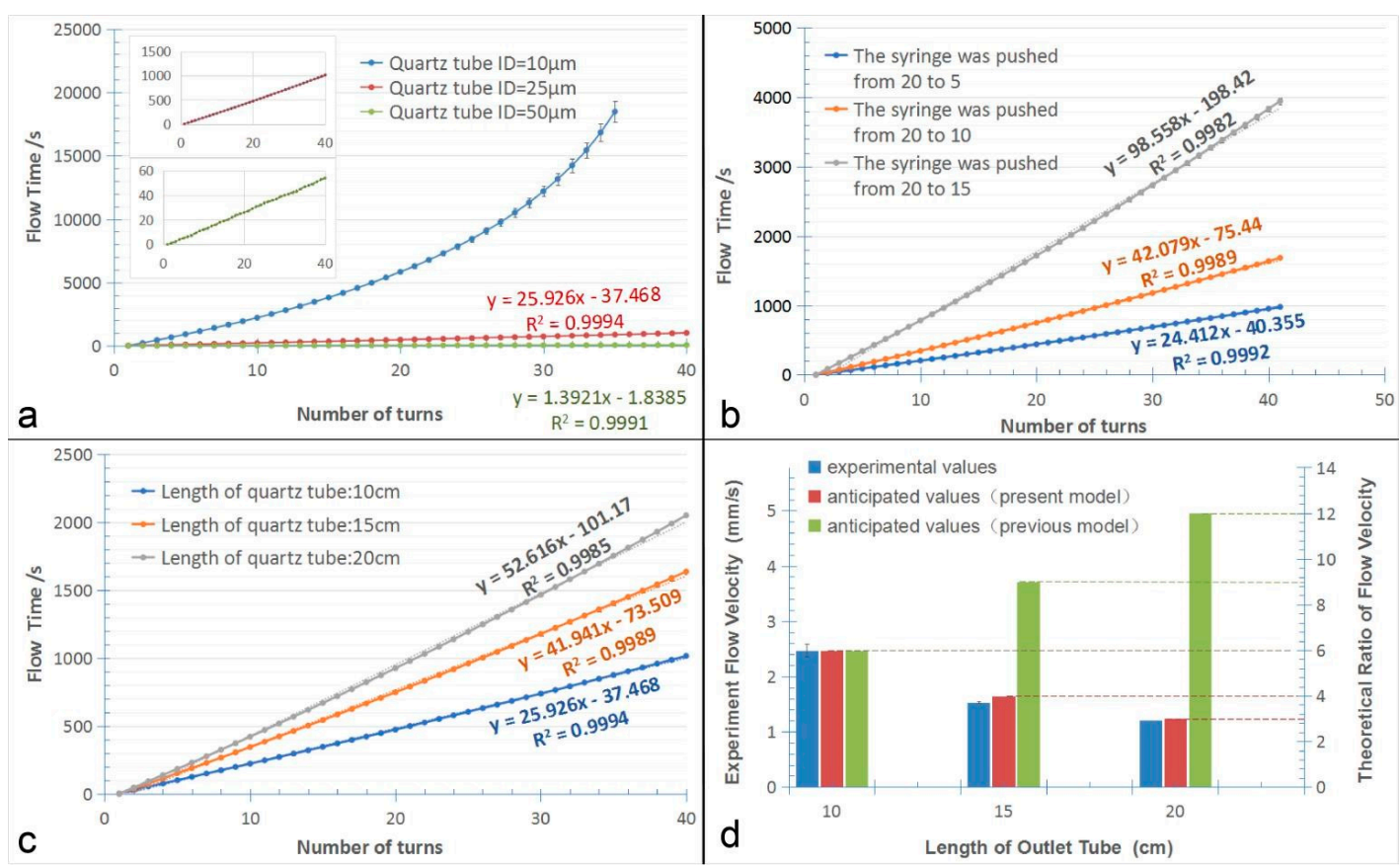

Figure 5. The self-activated flow influenced by (a) inner diameter of the tail quartz tube, (b) the pressure, and (c) the length of the tail quartz tube. (d) The relationship between the flow rates and the length of the tail quartz tube anticipated from two mechanisms, and the assays of measured flow velocities. (The previous model is based on our previous works [20,22-24], and the present model is our method, illustrated in this article.).

\subsection{Results of Plug-based Continuous Flow PCRs and Droplet-based Continuous Flow PCRs}

As a proof of concept, this novel micropump was firstly applied to plug-based continuous flow PCRs in the end-opened system. The Teflon tube wrapped PDMS block should be placed on the single heater for at least $30 \mathrm{~min}$ to reach the set temperature before sample injection. To make sure the surface temperatures corresponded to the annealing, extension and denature temperature of PCRs, the denature temperature was adjusted to approximately $95 \pm 0.8{ }^{\circ} \mathrm{C}(\mathrm{CV}=0.8 \%, \mathrm{n}=10)$, and the annealing temperature was adjusted to approximately $65 \pm 0.7^{\circ} \mathrm{C}(\mathrm{CV}=0.6 \%, \mathrm{n}=10)$.

The reaction condition in the commercial qPCR cycler is set as mentioned above, with the annealing stage and the denature stage set to be $30 \mathrm{~s}$ and $10 \mathrm{~s}$, respectively. Meanwhile, the flow velocity of the reagent in the Teflon tube wrapped PDMS block is approximately $42 \mathrm{~s}$ per cycle. Due to the shape of the PDMS block, the $95^{\circ} \mathrm{C}$ denature temperature lasted for $9 \mathrm{~s}$, and the $65^{\circ} \mathrm{C}$ annealing temperature lasted for $18 \mathrm{~s}$. Besides, the extension stage lasted for about $15 \mathrm{~s}$, which corresponds to the PDMS block side walls.

After the reaction, the reagents gained from the microdevice and qPCR cycler were tested by agarose gel electrophoresis. The results were shown in Figure 6. 

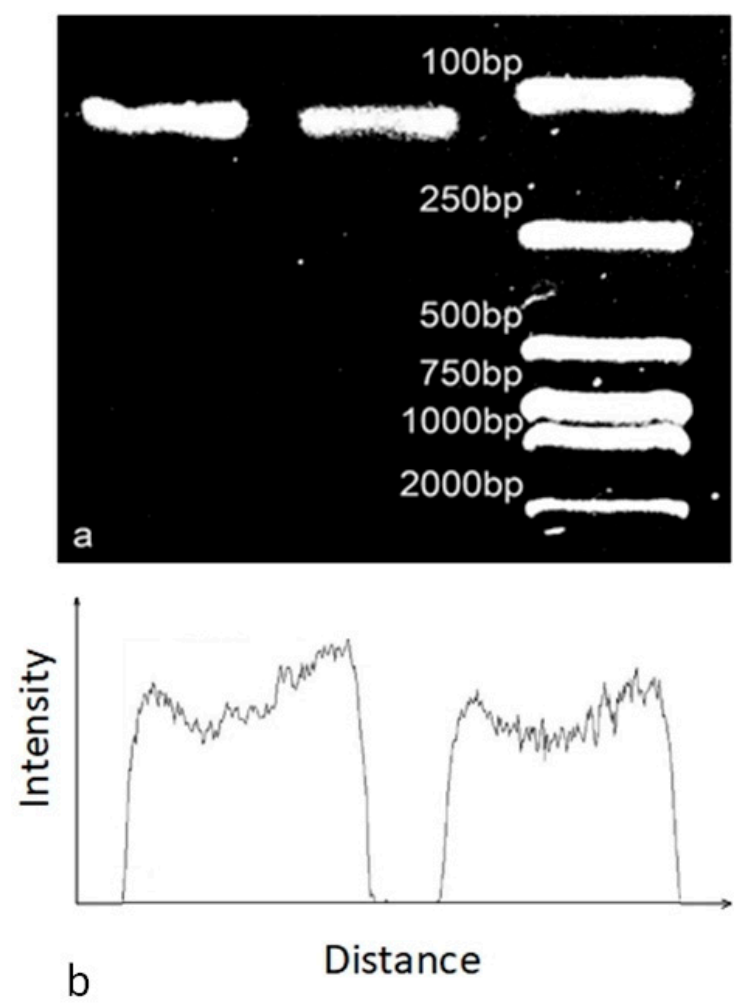

Figure 6. Electrophorogram and intensity diagram of contrast PCR experiments. (a) Electrophoretic results by commercial cycler (left), electrophoretic results by microdevice (middle) and the ladder (right). (b) The intensity diagram of commercial cycler PCR electrophoretic results (left) and microdevice PCR electrophoretic results (right).

The result showed in Figure 6 demonstrated that the plug-based amplification efficiency of the microdevice was similar to the commercial qPCR cycler. By calculating the average gel intensity of targets, the amplification efficiency of the microchip reached $85.7 \%$ of commercial qPCR cycler, which suggested this novel self-powered micropump could be applied to plug-based continuous flow PCRs and the results were similar to commercial qPCR devices.

This novel self-powered micropump was further applied to droplet-based continuous flow PCRs. The reaction condition in the commercial qPCR cycler is set the same as the plug-based experiment. Meanwhile, the flow rate of the reagent in the Teflon tube wrapped PDMS block is approximately $40 \mathrm{~s}$ per cycle.

Figure 7 demonstrated that droplets-based amplification efficiency of the microdevice was also similar to the commercial qPCR cycler. And the contrast amplification efficiency reached $91.5 \%$. We assumed that Fluorinated oil can keep the droplet away from the inner wall of the Teflon tube, which increased the efficiency of the reaction. 

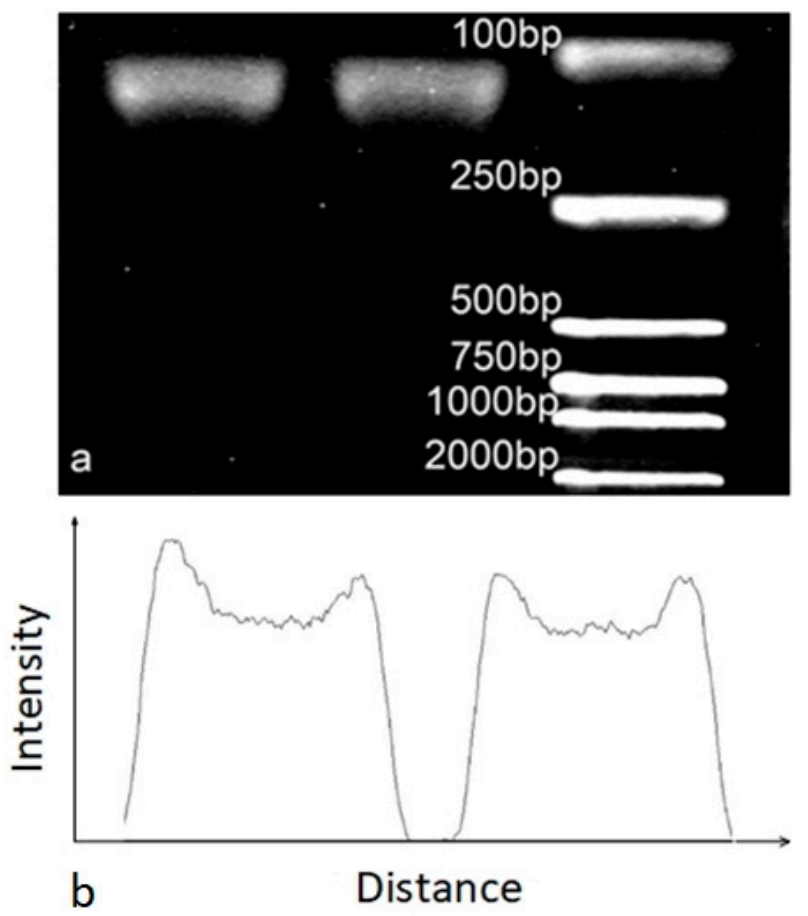

Figure 7. Electrophorogram and intensity diagram of the liquid droplets' PCR experiment. (a) Electrophoretic results by commercial cycler (left), electrophoretic results by microdevice (middle) and the ladder (right). (b) The intensity diagram of commercial cycler PCR electrophoretic results (left) and microdevice PCR electrophoretic results (right).

Based on the aforementioned flow analyses, it proved the passive micropump can stably transport both the single-phased and the double-phased liquid inside several meters' long microchannel of 3D configuration.

\subsection{Results of the Real-time Fluorescence Detection}

To find out the PCR amplification efficiency of the novel system, four ordered serially diluted genes from $10^{8}$ to $10^{5}$ copies $/ \mu \mathrm{L}$ were tested in both the micro-device and commercial qPCR cycler (Bio Rad). The images were obtained by digital camera (Canon EOS 7D). The results were shown in Figure 8.

As shown in Figure 8, dramatic decreased fluorescence signal could be seen as concentration of DNA template decreased from $10^{8}$ to $10^{5}$ copies $\mu \mathrm{L}^{-1}$. The fluorescence intensity curves shown in Figure 8a were obtained from camera images, and the software Image J was used to analyze fluorescence intensity of each image. The $\mathrm{Ct}$ values of the four curves were calculated to be 16.35, 19.22, 22.67 and 26.21. The results run by commercial qPCR were shown in Figure 8b, with the Ct values of $11.28,15.28,18.64$ and 20.98, respectively. According to Figure 8, we could find that the trend of the four curves in the former image were similar with the latter, which suggested that the novel micropump could be applied to self-activated continuous-flow and real-time PCRs, and the results were similar to commercial qPCR devices. 

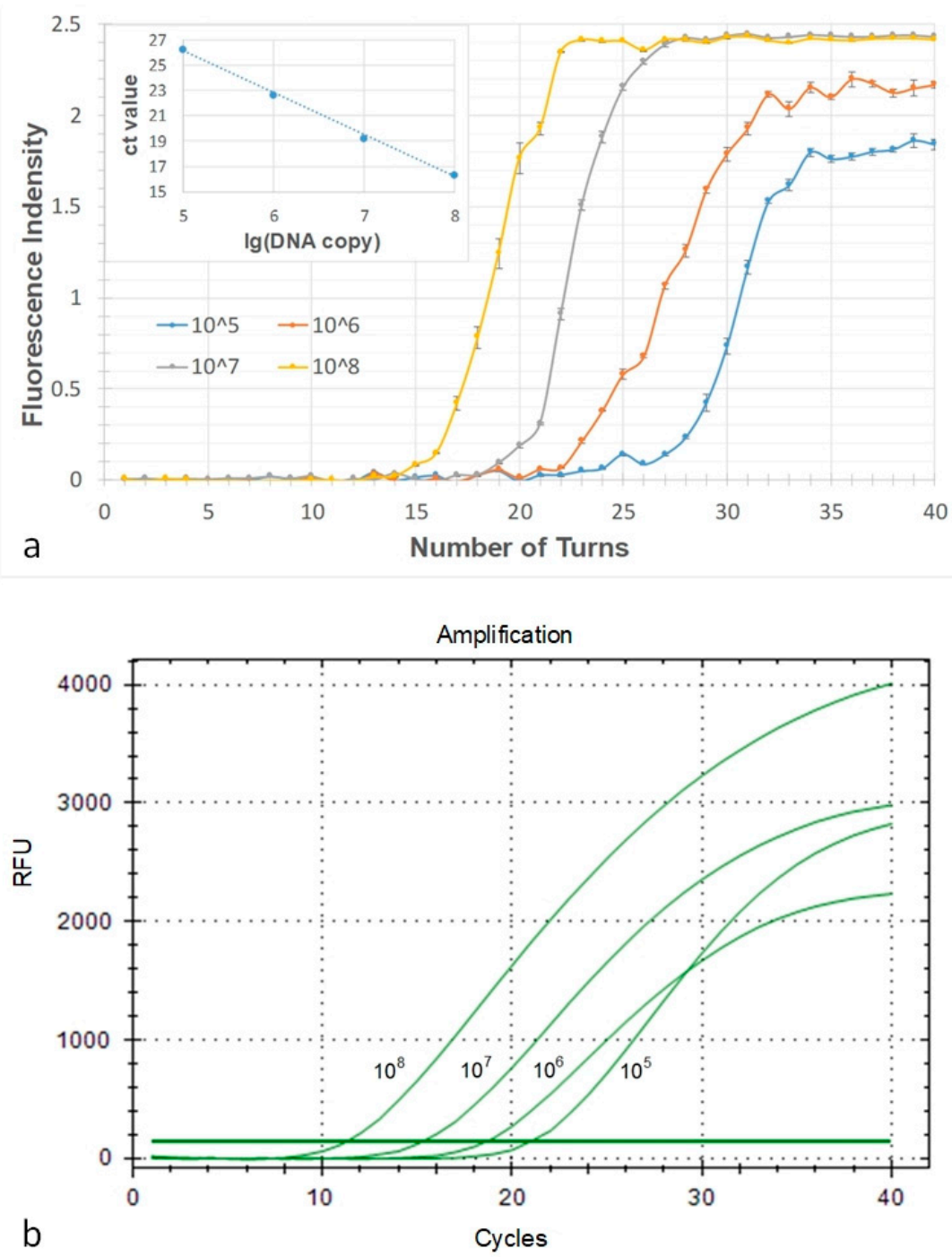

Figure 8. Results of real-time fluorescence detection. (a) The amplification curves gained from the fluorescence images of serial diluted DNA molecules of the microdevice. (b) Amplification curves from the commercial Real-time PCR amplification system.

\section{Conclusions}

We introduced a novel mechanism for self-powered liquid transport in the end-opened microchip. It provided a new approach to control the velocity of liquid conveniently by adopting the gas permeability of the quartz capillary. The flow assays confirmed a good coincidence between the theoretical formula and the experimental results. Furthermore, herein a totally contrary regularity with previous mechanisms [26-29] was also confirmed through the assays between the flow rate and the length of outlet-tube. In contrast with most other passive micropumps which have been developed until today, the passive micropump here displays the superior pumping performance concerning good bubbles' suppression under extreme microenvironment of high temperature $\left(95^{\circ} \mathrm{C}\right)$, and thus, capable of complicated applications such as the house-made setup of plug/droplet-based continuous-flow real-time on-chip PCRs. In future research, we are going to upgrade this system to make it more controllable, and to apply it to wider fields.

Author Contributions: Data curation, D.W.; formal analysis, D.W. and B.S.; validation, B.L.; writing-original draft, D.W.; writing — review \& editing, D.W.; conceptualization, W.W.; principle section, W.W.; funding acquisition, W.W.; supervision, W.W. 
Funding: This project is supported by National Natural Science Foundation of China (No. 61704169), the CAS Pioneer Hundred Talents Program, the Natural Science Foundation of Jilin Province (20180520112JH), the talent project of Jilin Province, and Fundamental Research Funds for the Central Universities.

Conflicts of Interest: The authors declare no conflict of interest.

\section{References}

1. Das, P.K.; Hasan, A.B.M.T. Mechanical micropumps and their applications: A review. In American Institute of Physics Conference Series; AIP Publishing: New York, NY, USA, 2017; pp. 917-942.

2. Qiu, X.; Chen, D.; Liu, C.; Mauk, M.G.; Kientz, T.; Bau, H.H. A portable, integrated analyzer for microfluidic-Based molecular analysis. Biomed. Microdevices 2011, 13, 809-817. [CrossRef] [PubMed]

3. Liu, J.; Guan, E.; Li, P.; Wang, F.; Liang, C.; Zhao, Y.; Liu, J.; Guan, E.; Li, P.; Wang, F. Deflection behavior of a piezo-driven flexible actuator for vacuum micropumps. Sens. Actuators A Phys. 2017, 267, 30-41. [CrossRef]

4. Schuhladen, S.; Banerjee, K.; Stürmer, M.; Müller, P.; Wallrabe, U.; Zappe, H. Variable optofluidic slit aperture. Light Sci. Appl. 2016, 5, e16005. [CrossRef] [PubMed]

5. Son, J.H.; Cho, B.; Hong, S.; Lee, S.H.; Hoxha, O.; Haack, A.J.; Lee, L.P. Ultrafast photonic PCR. Light Sci. Appl. 2015, 4, e280. [CrossRef]

6. Zhang, Z.; Kan, J.; Cheng, G.; Wang, H.; Jiang, Y. A piezoelectric micropump with an integrated sensor based on space-division multiplexing. Sens. Actuators A Phys. 2013, 6, 29-36. [CrossRef]

7. Eom, I.Y.; Niri, V.H.; Pawliszyn, J. Development of a syringe pump assisted dynamic headspace sampling technique for needle trap device. J. Chromatogr. A 2008, 1, 10-14. [CrossRef] [PubMed]

8. Go, U.J.; Eom, I.Y. Development of syringe pump assisted headspace sampler. J. Chromatogr. A 2014, 1361, 88-94. [CrossRef]

9. Li, Z.; Mak, S.Y.; Sauret, A.; Shum, H.C. Syringe-pump-induced fluctuation in all-aqueous microfluidic system implications for flow rate accuracy. Lab Chip 2014, 4, 744-749. [CrossRef]

10. Jun, D.H.; Yang, S.S. The influence of the geometry of the pump chamber on the characteristics of the thermopneumatic-drive surface tension micropump. Sens. Actuators A Phys. 2015, 3, 294-301. [CrossRef]

11. Ni, J.; Wang, B.; Chang, S.; Lin, Q. An integrated planar magnetic micropump. Microelectron. Eng. 2014, 4, 35-40. [CrossRef]

12. Hintermüller, M.A.; Jakoby, B.; Reichel, E.K. Numerical and experimental analysis of an acoustic micropump utilizing a flexible printed circuit board as an actuator. Sens. Actuators A Phys. 2017, 260, 220-227. [CrossRef]

13. Rimsa, R.; Smith, A.J.; Wälti, C.; Wood, C.D. A planar surface acoustic wave micropump for closed-loop microfluidics. Appl. Phys. Lett. 2017, 23, 234102. [CrossRef]

14. Yun, K.S.; Cho, I.J.; Bu, J.U.; Kim, C.J. A surface-tension driven micropump for low-voltage and low-power operations. Microelectromech. Syst. J. 2002, 5, 454-461.

15. Wu, W.; Manz, A. Rapid manufacture of modifiable 2.5-dimensional (2.5D) microstructures for capillary force-driven fluidic velocity control. RSC Adv. 2015, 87, 70737-70742. [CrossRef]

16. Xu, L.; Lee, H.; Oh, K.W. Syringe-assisted point-of-care micropumping utilizing the gas permeability of polydimethylsiloxane. Microfluid. Nanofluidics 2014, 17, 745-750. [CrossRef]

17. Choe, Y.; Kim, E.S. Valveless micropump driven by acoustic streaming. J. Micromech. Microeng. 2013, 4, 45005. [CrossRef]

18. Dutka, F.; Opalski, A.S.; Garstecki, P. Nano-liter droplet libraries from a pipette: Step emulsificator that stabilizes droplet volume against variation in flow rate. Lab Chip 2016, 11, 2044-2049. [CrossRef] [PubMed]

19. Trinh, K.T.L.; Wu, W.; Lee, N.Y. Fabrication of a 3D Teflon microdevice for energy free homogeneous liquid flow inside a long microchannel and its application to continuous-flow PCR. RSC Adv. 2017, 18, 10624. [CrossRef]

20. Henríquez, C.; Horstkotte, B.; Cerdà, V. A highly reproducible solenoid micropump system for the analysis of total inorganic carbon and ammonium using gas-diffusion with conductimetric detection. Talanta 2014, 118, 186-194. [CrossRef]

21. Xu, L.; Lee, H.; Jetta, D.; Oh, K.W. Vacuum-driven power-free microfluidics utilizing the gas solubility or permeability of polydimethylsiloxane (PDMS). Lab Chip 2015, 20, 3962-3979. [CrossRef] 
22. Wu, W.; Trinh, K.T.L.; Zhang, Y.; Lee, N.Y. Portable plastic syringe as a self-actuated pump for long-distance uniform delivery of liquid inside a microchannel and its application for flow-through polymerase chain reaction on chip. RSC Adv. 2015, 16, 12071-12077. [CrossRef]

23. Wu, W.; Trinha, K.T.L.; Lee, N.Y. Flow-through polymerase chain reaction inside a seamless 3D helical microreactor fabricated utilizing a silicone tube and a paraffin mold. Analyst 2015, 140, 1416-1420. [CrossRef] [PubMed]

24. Wu, W.; Trinh, K.T.; Lee, N.Y. Hand-held syringe as a portable plastic pump for on-chip continuous-flow PCR: Miniaturization of sample injection device. Analyst 2012, 4, 983-990. [CrossRef] [PubMed]

25. Jiang, Y.; Du, L.; Li, Y.; Mu, Q.; Cui, Z.; Zhou, J.; Wu, W. A novel mechanism for user-friendly and self-activated microdroplet generation capable of programmable control. Analyst 2018, 143, 3798-3807. [CrossRef] [PubMed]

26. Li, X.; Wu, W.; Manz, A. Thermal gradient for fluorometric optimization of droplet PCR in virtual reaction chambers. Microchim. Acta 2017, 9, 1-7. [CrossRef]

27. Qin, L.; Vermesh, O.; Shi, Q.; Heath, J.R. Self-powered microfluidic chips for multiplexed protein assays from whole blood. Lab Chip 2009, 14, 2016-2020. [CrossRef] [PubMed]

28. Wu, W.; Kang, K.T.; Lee, N.Y. Bubble-free on-chip continuous-flow polymerase chain reaction: Concept and application. Analyst 2011, 11, 2287-2293. [CrossRef]

29. Wu, W.; Lee, N.Y. Two-layer microdevice for parallel flow-through PCRs employing plastic syringes for semi-automated sample injection and a single heater for amplification: Toward process simplification and system miniaturization. Sens. Actuators B Chem. 2013, 181, 756-765. [CrossRef]

(C) 2019 by the authors. Licensee MDPI, Basel, Switzerland. This article is an open access article distributed under the terms and conditions of the Creative Commons Attribution (CC BY) license (http://creativecommons.org/licenses/by/4.0/). 